\title{
Anatomia do xilema secundário de sete espécies do gênero Tachigali Aubl. (Fabaceae), disponíveis na Xiloteca Walter A. Egler, do Museu Paraense Emílio Goeldi, Pará, Brasil The anatomy of the secondary xylem of seven species of the Tachigali Aubl. type. from the Walter A. Egler wood collection of the Museu Paraense Emílio Goeldi, Pará, Brazil
}

\author{
Alisson Rodrigo Souza Reis', Pamella Carolline Marques dos Reis", \\ Alcir Tadeu de Oliveira Brandão"I , Pedro Luiz Braga Lisboa III \\ Universidade Federal do Pará. Altamira, Pará, Brasil \\ "Universidade Federal Rural da Amazônia. Belém, Pará, Brasil \\ IIIMuseu Paraense Emílio Goeldi. Coordenação de Botânica. Belém, Pará, Brasil
}

\begin{abstract}
Resumo: As espécies de Tachigali Aubl. apresentam potencial para reflorestamentos e produção de energia. Entretanto, informações de características ainda são incipientes, dificultando a identificação dessas madeiras. O objetivo do trabalho foi caracterizar anatomicamente sete espécies de Tachigali, disponíveis na xiloteca Walter A. Egler, do Museu Paraense Emílio Goeldi, como contribuição à identificação das seguintes espécies: T. froesii (Pires) L.F. Gomes da Silva \& H.C. Lima, T. melanocarpa (Ducke) Van DerWeff, T. paniculata Aubl., T. paraensis (Huber) Barneby, T. reticulosa (Dwyer) Zarucchi \& Herend., T. setifera (Ducke)Zarucchi \& Herend e T. tinctoria Bent. var. tinctoria (Benth.) Zarucchi \& Herend. As análises seguiram as metodologias tradicionais em anatomia de madeira e microscopia eletrônica de varredura (MEV). As espécies apresentam raios unisseriados e homogêneos, com exceção de T. froesii e T. melanocarpa, na qual são unisseriados e heterogêneos. O parênquima paratraqueal vasicêntrico está presente em todas as espécies, associado com outro tipo de parênquima paratraqueal. Os poros são predominantemente solitários, exceto em T. melanocarpa e T. reticulosa, com poros múltiplos na maioria dos casos. Tachigali froesii foi a única espécie que apresentou floema incluso e cristais de carbonato de cálcio. A composição e a organização das células do raio, aliadas ao tipo de parênquima axial, revelam a peculiaridade de cada espécie.
\end{abstract}

Palavras-chave: Anatomia da madeira. Tachigali Aubl. Caesalpinioideae. Leguminosae.

\begin{abstract}
Species of Tachigali Aubl. have potential for reforestation and biofuel projects. However, information about the wood anatomy of this species is still very embryonic, hampering identification work. The objective of this study was to anatomically characterize seven species of Tachigali available in the Walter. A. Egler wood collection of the Museu Paraense Emilio Goeldi, thereby facilitating future identification work. The following species were studied: T. froesii (Pires) L.F. Gomes da Silva \& H.C. Lima, T. melanocarpa (Ducke) Van Der Weff, T. paniculata Aubl., T. paraensis (Huber) Barneby, T. reticulosa (Dwyer) Zarucchi \& Herend., T. setifera (Ducke) Zarucchi \& Herend e T. tinctoria Bent. var. tinctoria (Benth.) Zarucchi \& Herend. Analyses followed standard wood anatomy techniques and scanning electronic microscopy (SEM). All species except T. froesii and T. melanocarpa present uniseriate and homogeneous rays, with the exceptions being heterogeneous. A vasicentric paratraqueal parenchyma is present in all the species, combined with another type of parenchyma. The pores are predominantly solitary, except in T. melanocarpa and T. reticulosa which have multiple pores. Tachigali froesii was the only species that presented enclosed bast and calcium carbonate crystals. The composition and organization of the ray cells, allied to the axial type of parenchyma, is important in distinguishing the peculiarity of each species.
\end{abstract}

Keywords: Wood anatomy. Tachigali Aubl. Caesalpinioideae, Leguminosae.

REIS, A. R. S., P. C. M. REIS, A. T. O. BRANDÃO \& P. L. B. LISBOA, 2011. Anatomia do xilema secundário de sete espécies do gênero Tachigali Aubl. (Fabaceae), disponíveis na Xiloteca Walter A. Egler do Museu Paraense Emílio Goeldi, Pará, Brasil. Boletim do Museu Paraense Emílio Goeldi. Ciências Naturais 6(3): 319-333.

Autor para correspondência: Alisson Rodrigo Souza Reis. Universidade Federal do Pará. Campus de Altamira. Rua Coronel José Porfírio, 2515 - São Sebastião. Altamira, PA, Brasil. CEP 68372-000 (alissonreis@ufpa.br).

Recebido em 12/04/2010

Aprovado em 03/01/2012

Responsabilidade editorial: Ana Cristina Andrade de Aguiar Dias

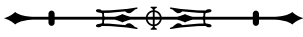




\section{INTRODUÇÃO}

"Nos últimos anos, o crescimento populacional, o avanço tecnológico e a crise dos combustíveis fósseis aumentaram a pressão sobre a flora nativa de muitas regiões em diferentes partes do mundo, incluindo o Brasil, nas mais variadas formas, com destaque para a produção de lenha e de carvão vegetal" (Oliveira et al., 2006).

Nesse contexto, o reflorestamento de espécie nativas de rápido crescimento para a produção de energia torna-se uma opção viável, como é o caso de espécies de Tachigali Aubl. (leguminosae), que possui indivíduos de crescimento rápido, com elevada produção de fitomassa e desrama foliar, o que possibilita uma rápida formação de serrapilheira, podendo ser usado para recuperação de áreas degradadas, reflorestamentos e para produção de energia.

Após a realização de vários trabalhos taxonômicos, como os de Lewis et al. (2005), Graham \& Barker (1981), Haston et al. (2003, 2005) e Silva \& Lima (2007), o gênero Sclerolobium Vogel. tornou-se sinônimo de Tachigali Aubl. Este trabalho utilizará esta classificação, realizando levantamento bibliográfico dos táxons até então distintos.

Apesar da escassez de estudos sobre Tachigali, vários trabalhos de anatomia da madeira vêm sendo realizados em espécies de Leguminosae, devido ao fato de a família ter grande importância econômica, principalmente na região amazônica, além de ser a terceira maior família das angiospermas.

Os trabalhos estão voltados, principalmente, para a anatomia sistemática, como é o caso de Bareta-Kuipers (1981), que relacionou característica anatômica com a filogenia das subfamílias e também em nível de tribo e gênero; Angyalossy-Alfonso \& Miller (2002) caracterizaram espécies de Swartzia e considerações sobre a tribo Swartzieae; Gasson et al. (2004) discorreram sobre as relações dos caracteres anatômicos da tribo Millettieae com a Papilonoideae; Gasson \& Wray (2001) descreveram e sistematizaram Cyathostegia mathewsii; Gasson (1999) realizou o mesmo estudo com a tribo Dipterygeae. Höhn (1999) comparou madeiras arqueológicas das subfamílias
Caesalpinioideae e Mimosoideae ocorrentes nas savanas do leste da África; já Pernía \& Melandri (2006) compararam madeiras da tribo Caesalpinieae da Venezuela.

Outra linha de pesquisas anatômicas da família é a determinação de espécies, como é o caso de Loureiro et al. (1983, 1997), que caracterizam quatro espécies de Caesalpiniaceae, incluindo Sclerolobium chrysophyllum Poepp. \& Endl. Loureiro et al. (2000) descreveram as características gerais e anatômicas de diversas espécies de leguminosas da Amazônia, entre estas Sclerolobium paraense Huber. Já Pires \& Marcati (2005) estudaram a anatomia e uso da madeira de duas variedades de Sclerolobium paniculatum Vog. do sul do Maranhão, Brasil.

Ferreira et al. (2004) e Ferreira \& Hopkins (2004) realizaram estudo anatômico das espécies de Leguminosae comercializadas no estado do Pará como "Angelim". Marchiori (2007) estudou a dendrologia de diversas espécies de leguminosas, entre elas vários gêneros da subfamília Caesalpinioideae. Já Silva et al. (2009) caracterizaram a anatomia e densidade básica do tronco e de galhos de Caesalpinia pyramidalis Tul., espécie endêmica da caatinga com potencial para a fabricação de carvão.

Sabendo da importância da família, em específico do gênero Tachigali, aliado ao grande número de espécies que são utilizadas pela indústria madeireira, a anatomia da madeira torna-se a principal ferramenta utilizada para a identificação, auxiliando, principalmente, os órgãos de fiscalização.

Com isso, é fundamental que na região amazônica, onde há um grande contingente de madeiras sendo exploradas, exista um banco de dados com informações anatômicas, além do armazenamento de madeiras para a identificação correta dessas espécies.

As xilotecas brasileiras contêm acervos com uma significativa coleção de madeiras das florestas existentes no Brasil e no exterior. Na região amazônica, apesar do grande número de madeiras comercializadas, existem apenas três grandes coleções, uma das quais é a da Xiloteca Walter A. Egler, do Museu Paraense Emílio Goeldi, criada em 1979, onde estão depositadas cerca de 7.300 amostras 
de madeiras, basicamente amazônicas, que serviu de base para esse estudo. Este trabalho objetivou caracterizar o xilema secundário de sete espécies amazônicas de interesses comerciais, pertencentes ao gênero Tachigali Aubl. (Leguminosae), com potencial para reflorestamento e produção de carvão e lenha.

\section{MATERIAL E METODOS}

\section{AMOSTRAGEM E PREPARO DO MATERIAL}

As amostras foram obtidas na xiloteca Walter A. Egler, do Museu Paraense Emílio Goeldi, com repetições, quando disponíveis (Tabela 1). Todas as amostras estão devidamente identificadas e o material testemunha incorporado no herbário da instituição (MG). Em laboratório, foram retirados corpos de prova $(2 \times 2 \times 3 \mathrm{~cm})$, orientados nos planos transversal, longitudinal radial e tangencial para a descrição macro e microscópica.

\section{ANÁLISE DA ESTRUTURA MACROSCÓPICA DO LENHO}

Para a descrição das superfícies transversais e longitudinais do lenho, os corpos de prova foram polidos para melhor visualização das estruturas celulares. As estruturas anatômicas foram visualizadas sob lupa conta-fio com aumento de dez vezes e estereomicroscópio Motic, sendo descritas conforme as normas de Coradin \& Muniz (1992).

As imagens macroscópicas foram obtidas da superfície transversal do corpo de prova, utilizando-se de um estereomicroscópio Motic acoplado ao microcomputador, com auxílio do software Motic plus 2.0.

\section{ANÁLISE DA ESTRUTURA MICROSCÓPICA DO LENHO}

\section{Microscopia óptica}

Os corpos de prova das sete espécies de Tachigali foram imersos e fervidos em água destilada para amolecimento e, em seguida, seccionados em micrótomo de deslize na espessura de 15-20 $\mu \mathrm{m}$, nos planos transversal, longitudinal tangencial e longitudinal radial. Estas secções foram clarificadas com hipoclorito de sódio 10\%, lavadas em água destilada, desidratadas até etanol 50\%, coradas com safranina alcoolica $1 \%$, desidratadas em série alcoolica etanol-butílica, selecionadas e montadas em lâmina de vidro com bálsamo do Canadá.

Para o processo de maceração, segmentos do lenho foram transferidos para tubos de ensaio com água destilada e, em seguida, com solução macerante (ácido acético glacial e água oxigenada 120 volumes), levados à estufa a $60{ }^{\circ} \mathrm{C}$ por 24 horas, escoados e lavados com água corrente (Franklin, 1945). Para o estudo da morfologia e a avaliação das dimensões, as células dissociadas do lenho foram coradas com safranina aquosa a $1 \%$ e montadas em lâminas semipermanentes com glicerina, visualizadas sob microscópio Leica, acoplado à câmera de vídeo Sony e sistema de análise de imagens, com auxílio do software Visilog 5.1., no qual foram mensuradas 50 unidades de fibras e elementos de vaso, obtendo-se o comprimento, espessura da parede e largura do lume das fibras, o comprimento e o diâmetro dos elementos de vasos em micras $(\mu \mathrm{m})$, além do comprimento e largura dos raios na mesma unidade. Para as contagens de comprimento e largura dos raios em número de células e em micras $(\mu \mathrm{m})$, foram obtidas imagens a partir de cortes histológicos permanentes e semipermanentes, sendo adquiridas por meio do software Aver TV.

A descrição da estrutura microscópica do lenho das espécies foi conduzida de acordo com as normas da IAWA Committee (1989).

\section{Microscopia Eletrônica de Varredura}

Para este procedimento, seguiram-se as recomendações de Silveira (1989, com modificações). Foram selecionados cortes histológicos com espessura entre 20-25 $\mu \mathrm{m}$, os quais foram desidratados em série etílica e butílica. As amostras foram então aderidas a lâminas de vidro, com 
Tabela 1. Lista de espécies estudadas e depositadas na xiloteca Walter A. Egler, do Museu Paraense Emílio Goeldi.

\begin{tabular}{|c|c|c|c|c|}
\hline Espécie & Procedência & Coletor & $\begin{array}{l}\text { Número de } \\
\text { coleta }\end{array}$ & Registro \\
\hline $\begin{array}{l}\text { Tachigali froesii (Pires) L.F. Gomes } \\
\text { da Silva \& H.C. Lima }\end{array}$ & $\begin{array}{c}\text { Inventário entre Porto Platon } \\
\text { e Serra do Navio }\end{array}$ & Rosa, N. A. & 1401 & $\times 1217$ \\
\hline $\begin{array}{l}\text { Tachigali froesii (Pires) L.F. Gomes } \\
\text { da Silva \& H.C. Lima Pires }\end{array}$ & Bosquinho & Silva, M. G. & 4266 & $\times 2058$ \\
\hline $\begin{array}{l}\text { Tachigali melanocarpa (Ducke) } \\
\text { Van Der Werff }\end{array}$ & $\begin{array}{c}\text { Inventário entre Porto Platon } \\
\text { e Serra do Navio }\end{array}$ & Rosa, N. A. & 1388 & $\times 1198$ \\
\hline $\begin{array}{l}\text { Tachigali melanocarpa (Ducke) Van } \\
\text { Der Werff }\end{array}$ & $\begin{array}{c}\text { Companhia Floresta Monte } \\
\text { Dourado, Jarí }\end{array}$ & Silva, N. T. & 5144 & $\times 5514$ \\
\hline Tachigali paniculata Aubl. & $\begin{array}{l}\text { Tucuruí, próximo ao rio Macoari, } \\
\text { margem direita do rio Tocantins }\end{array}$ & $\begin{array}{c}\text { Marciel, U. N.; } \\
\text { Silva, M. G. e } \\
\text { Rosário, C. }\end{array}$ & 665 & $\times 3289$ \\
\hline Tachigali paraensis (Huber) Barneby & Monte Dourado, área do Munguba & Silva, N. T. & 5451 & $\times 5265$ \\
\hline $\begin{array}{l}\text { Tachigali reticulosa (Dwyer) Zarucchi } \\
\text { \& Herend. }\end{array}$ & Margem direita do rio Caruauna & $\begin{array}{l}\text { Rosário, C. e } \\
\text { Cardoso, O. N. }\end{array}$ & 832 & $\times 6127$ \\
\hline $\begin{array}{l}\text { Tachigali setifera (Ducke) Zarucchi } \\
\text { \& Herend }\end{array}$ & $\begin{array}{c}\text { Presidente Médici, estrada para } \\
\text { Costa Marques, próximo à Alvorada }\end{array}$ & $\begin{array}{c}\text { Silva, M. G.; } \\
\text { Santos, M. R. e } \\
\text { Silva, C. R. }\end{array}$ & 6177 & $\times 3982$ \\
\hline $\begin{array}{l}\text { Tachigali tinctoria Benth. var. tinctoria } \\
\text { (Benth.) Zarucchi \& Herend. }\end{array}$ & Bacia do Jarí & Silva, N. T. & 5115 & $\times 5938$ \\
\hline
\end{tabular}

auxílio de fita dupla face, metalizadas com $20 \mathrm{~nm}$ de ouro, observadas e documentadas em microscópio eletrônico de varredura (MEV) Leo modelo 1450 VP em aceleração de voltagem entre 10 e $15 \mathrm{Kv}$. Para a identificação dos elementos químicos constituintes dos cristais, foi utilizado detector de raio-X (EDS) acoplado ao MEV.

\section{RESULTADOS}

\section{TACHIGALI FROESII (PIRES) L.F. GOMES DA SILVA \& H.C. LIMA}

Descrição macroscópica (Figura 1A): parênquima axial contrastado, visível somente sob lente de dez vezes, vasicêntrico e unilateral, formando arranjos diagonais. Raios notados a olho nu no topo e na face tangencial, não estratificado. Vasos notados a olho nu, sendo a maioria solitária. Camadas de crescimento individualizadas por zonas fibrosas tangenciais mais escuras.
Descrição microscópica: porosidade difusa, vasos solitários, ocorrendo também múltiplos de dois, três e quatro (Figura 2A); diâmetro variando de 148,80 até $348,63 \mu \mathrm{m}$; forma da seção arredondada, pontoações parênquimo-vasculares alternas, até cinco vasos por $\mathrm{mm}^{2}$; elementos vasculares de 175,94 a 498,15 $\mu \mathrm{m}$ de comprimento (Tabela 2); fibras (Figura 3A) com pontoações simples, não septadas, com paredes muito delgadas, variando de 492,70 até 877,26 $\mu \mathrm{m}$ de comprimento; diâmetro do lume com 9,21 até 44,05 $\mu \mathrm{m}$; espessura da parede de 2,58 a 6,28 $\mu$ m (Tabela 3). Parênquima axial paratraqueal predominantemente vasicêntrico e raramente unilateral. Raios não estratificados e heterogêneos, com células procumbentes e células quadradas nas extremidades (Figura 4A), apresentando altura em número de células de três a 14, variando de 137,78 até $372,59 \mu \mathrm{m}$ de altura; largura em número de células 1 , sendo 100\% unisseriado e 17,58 a 40,52 $\mu \mathrm{m}$ 
Tabela 2. Dados quantitativos de vasos/poros das espécies estudadas. Dp - desvio padrão.

\begin{tabular}{|c|c|c|c|c|c|c|c|c|c|c|c|c|}
\hline \multirow{2}{*}{ Espécie } & \multicolumn{4}{|c|}{ Comprimento $(\mu \mathrm{m})$} & \multicolumn{4}{|c|}{ Diâmetro $(\mu \mathrm{m})$} & \multicolumn{4}{|c|}{ Número de poros/mm } \\
\hline & Mín. & Méd. & Máx. & Dp & Mín. & Méd. & Máx. & Dp & Mín. & Méd. & Máx. & $\mathrm{Dp}$ \\
\hline $\begin{array}{l}\text { Tachigali froesii (Pires) L.F. } \\
\text { Gomes da Silva \& H.C. Lima }\end{array}$ & 175,94 & 340,49 & 498,15 & 85,24 & 148,80 & 221,49 & 348,63 & 48,82 & 1,00 & 3,20 & 5,00 & 0,92 \\
\hline $\begin{array}{l}\text { Tachigali melanocarpa (Ducke) } \\
\text { Van Der Weff. }\end{array}$ & 200,72 & 373,88 & 517,37 & 96,25 & 117,24 & 234,04 & 876,03 & 147,21 & 4,00 & 5,84 & 7,00 & 0,94 \\
\hline Tachigali paniculata Aubl. & 113,20 & 287,07 & 476,50 & 94,27 & 137,33 & 287,72 & 585,68 & 112,93 & 3,00 & 4,76 & 7,00 & 0,88 \\
\hline $\begin{array}{l}\text { Tachigali paraensis (Huber) } \\
\text { Barneby }\end{array}$ & 220,80 & 354,47 & 485,30 & 79,36 & 156,20 & 219,53 & 313,81 & 36,95 & 3,00 & 4,20 & 6,00 & 1,11 \\
\hline $\begin{array}{l}\text { Tachigali reticulosa (Dwyer) } \\
\text { Zarucchi \& Herend. }\end{array}$ & 229,00 & 320,32 & 466,01 & 93,01 & 120,71 & 168,56 & 231,85 & 30,30 & 6,00 & 8,72 & 13,00 & 2,28 \\
\hline $\begin{array}{l}\text { Tachigali setifera (Ducke) } \\
\text { Zarucchi \& Herend. }\end{array}$ & 214,33 & 343,93 & 550,25 & 50,35 & 118,48 & 225,43 & 416,97 & 39,65 & 3,00 & 4,08 & 6,00 & 0,99 \\
\hline $\begin{array}{l}\text { Tachigali tinctoria Benth. var. } \\
\text { tinctora(Benth.) Zarucchi } \\
\text { \& Herend. }\end{array}$ & 229,70 & 327,48 & 428,30 & 57,59 & 118,90 & 201,29 & 267,30 & 39,54 & 3,00 & 3,76 & 6,00 & 1,01 \\
\hline
\end{tabular}

Tabela 3. Dados quantitativos das fibras das espécies estudadas. Dp - desvio padrão.

\begin{tabular}{|c|c|c|c|c|c|c|c|c|c|c|c|c|}
\hline \multirow{2}{*}{ Espécie } & \multicolumn{4}{|c|}{ Comprimento $(\mu \mathrm{m})$} & \multicolumn{4}{|c|}{ Diâmetro do lume $(\mu \mathrm{m})$} & \multicolumn{4}{|c|}{ Espessura da parede $(\mu \mathrm{m})$} \\
\hline & Mín. & Méd. & Máx. & Dp & Mín. & Méd. & Máx. & $\mathrm{Dp}$ & Mín. & Méd. & Máx. & Dp \\
\hline $\begin{array}{l}\text { Tachigali froesii (Pires) L.F. } \\
\text { Gomes da Silva \& H.C. Lima }\end{array}$ & 492,70 & 690,41 & 877,26 & 78,56 & 9,21 & 17,45 & 44,05 & 5,39 & 2,58 & 3,95 & 6,28 & 0,77 \\
\hline $\begin{array}{l}\text { Tachigali melanocarpa (Ducke) } \\
\text { Van Der Weff. }\end{array}$ & 526,49 & 758,72 & 975,35 & 108,09 & 7,70 & 13,66 & 22,61 & 3,45 & 3,25 & 4,52 & 6,18 & 0,68 \\
\hline Tachigali paniculata Aubl. & 440,73 & 651,25 & 902,36 & 111,83 & 10,00 & 13,78 & 17,39 & 1,64 & 2,24 & 3,74 & 5,87 & 0,68 \\
\hline $\begin{array}{l}\text { Tachigali paraensis (Huber) } \\
\text { Barneby }\end{array}$ & 631,50 & 1062,64 & 1403,30 & 169,91 & 9,45 & 17,52 & 33,93 & 4,90 & 2,58 & 3,61 & 5,26 & 0,65 \\
\hline $\begin{array}{l}\text { Tachigali reticulosa (Dwyer) } \\
\text { Zarucchi \& Herend. }\end{array}$ & 580,18 & 738,76 & 1022,71 & 101,89 & 11,42 & 15,18 & 19,89 & 1,95 & 2,14 & 3,04 & 4,49 & 0,58 \\
\hline $\begin{array}{l}\text { Tachigali setifera (Ducke) } \\
\text { Zarucchi \& Herend. }\end{array}$ & 644,20 & 849,18 & 1220,52 & 124,88 & 7,10 & 12,93 & 17,72 & 2,20 & 2,41 & 3,57 & 5,08 & 0,61 \\
\hline $\begin{array}{l}\text { Tachigali tinctoria Benth. var. } \\
\text { tinctoria(Benth.) Zarucchi } \\
\text { \& Herend. }\end{array}$ & 501,22 & 746,79 & 960,11 & 116,92 & 11,42 & 15,91 & 21,67 & 2,97 & 1,00 & 3,09 & 4,74 & 0,76 \\
\hline
\end{tabular}

de largura; número de raios por milímetro linear variando de 7 a 12 raios (Tabela 4). Floema incluso (Figura 5F) presente. Cristais de carbonato de cálcio detectados por EDS/MEV (Figura 5D) na forma prismática, presentes nas células procumbentes dos raios (Figura 5C).

\section{TACHIGALI MELANOCARPA (DUCKE) VAN DER} WERFF

Descrição macroscópica (Figura 1B): parênquima axial contrastado, visível somente sob lente de dez vezes, aliforme confluente e raramente vasicêntrico, formando 
Tabela 4. Dados quantitativos de raios das espécies estudadas. Dp - desvio padrão.

\begin{tabular}{|c|c|c|c|c|c|c|c|c|c|c|c|c|c|c|c|c|c|c|c|c|}
\hline \multirow{2}{*}{ Espécie } & \multicolumn{4}{|c|}{ Altura $(\mu \mathrm{m})$} & \multicolumn{4}{|c|}{ Altura (célula) } & \multicolumn{4}{|c|}{ Largura $(\mu \mathrm{m})$} & \multicolumn{4}{|c|}{ Largura (célula.) } & \multicolumn{4}{|c|}{ Raios/mm } \\
\hline & Mín. & Méd. & Máx. & Dp & Mín. & Méd. & Máx. & Dp & Mín. & Méd. & Máx. & Dp & Mín. & Méd. & Máx. & Dp & Mín. & Méd. & Máx. & $D p$ \\
\hline $\begin{array}{l}\text { Tachigali froesii (Pires) L.F. } \\
\text { Gomes da Silva \& H.C. Lima }\end{array}$ & 137,78 & 208,40 & 372,59 & 50,66 & 3,00 & 7,22 & 14,00 & 2,53 & 17,58 & 26,89 & 40,52 & 5,13 & 1,00 & 1,00 & 1,00 & 0,00 & 7,00 & 9,78 & 12,00 & 1,42 \\
\hline $\begin{array}{l}\text { Tachigali melanocarpa } \\
\text { (Ducke) Van Der Weff. }\end{array}$ & 89,28 & 185,56 & 280,62 & 41,78 & 7,00 & 9,62 & 14,00 & 1,30 & 7,60 & 24,02 & 42,70 & 5,66 & 1,00 & 1,08 & 2,00 & 0,30 & 8,00 & 9,88 & 13,00 & 1,30 \\
\hline Tachigali paniculata Aubl. & 118,56 & 187,60 & 258,92 & 31,12 & 9,00 & 12,84 & 16,00 & 2,23 & 17,45 & 16,18 & 27,35 & 7,46 & 1,00 & 1,12 & 2,00 & 0,33 & 8,00 & 10,44 & 14,00 & 1,60 \\
\hline $\begin{array}{l}\text { Tachigali paraensis (Huber) } \\
\text { Barneby }\end{array}$ & 127,55 & 210,98 & 406,73 & 58,18 & 8,00 & 15,58 & 20,00 & 3,72 & 7,54 & 19,07 & 30,15 & 5,54 & 1,00 & 1,00 & 1,00 & 0,00 & 9,00 & 10,80 & 15,00 & 1,50 \\
\hline $\begin{array}{l}\text { Tachigali reticulosa (Dwyer) } \\
\text { Zarucchi \& Herend. }\end{array}$ & 71,13 & 138,59 & 280,26 & 41,32 & 3,00 & 6,74 & 14,00 & 2,40 & 9,39 & 15,96 & 23,25 & 3,23 & 1,00 & 1,08 & 2,00 & 0,20 & 6,00 & 8,10 & 10,00 & 1,22 \\
\hline $\begin{array}{l}\text { Tachigali setifera (Ducke) } \\
\text { Zarucchi \& Herend. }\end{array}$ & 74,42 & 128,90 & 215,55 & 27,09 & 2,00 & 9,00 & 15,00 & 2,56 & 8,25 & 14,14 & 26,80 & 3,75 & 1,00 & 1,00 & 1,00 & 0,00 & 6,00 & 8,48 & 12,00 & 1,44 \\
\hline $\begin{array}{l}\text { Tachigali tinctoria Benth. } \\
\text { var. tinctoria(Benth.) } \\
\text { Zarucchi \& Herend. }\end{array}$ & 70,60 & 114,23 & 194,30 & 25,11 & 3,00 & 6,54 & 10,00 & 1,96 & 10,10 & 14,47 & 20,20 & 2,41 & 1,00 & 1,00 & 1,00 & 0,00 & 6,00 & 8,58 & 11,00 & 1,42 \\
\hline
\end{tabular}

arranjos tangenciais. Raios visíveis somente sob lente de dez vezes, não estratificados. Vasos visíveis somente sob lente de dez vezes, múltiplos em sua maioria. Camadas de crescimento indistintas.

Descrição microscópica: porosidade difusa, vasos múltiplos, ocorrendo também solitários (Figura 2B); diâmetro tangencial de 117,24 a 876,03 $\mu \mathrm{m}$; forma da seção arredondada, pontoações intervasculares alternas; até sete vasos por $\mathrm{mm}^{2}$; elementos vasculares variando de 200,72 a 517,37 $\mu$ m de comprimento (Tabela 2). Fibras (Figura 3B) com pontoações simples, não septadas, com paredes muito finas, variando de 526,49 a 975,35 $\mu$ m de comprimento; diâmetro do lume de 7,70 a 22,61 $\mu$ m; espessura da parede de 3,25 a 6,18 $\mu \mathrm{m}$ (Tabela 3). Parênquima axial paratraqueal aliforme confluente e raramente vasicêntrico. Raios não estratificados e heterogêneos, constituídos de filas de células procumbentes e filas de células quadradas que se alternam (Figura 4B), altura em número de células variando de 7 a 14 e 89,28 a 280,62 $\mu$ m de altura; largura em número de células 1 a 2, sendo predominantemente (90\%) unisseriado, variando de 7,60 a 42,70 $\mu \mathrm{m}$ de largura; número de raios por milímetro linear variando de 8 a 13 raios (Tabela 4).

\section{TACHIGALI PANICULATA AUBL.}

Descrição macroscópica (Figura 1C): parênquima axial contrastado, visível somente sob lente de dez vezes, aliforme e vasicêntrico, formando arranjos diagonais. Raios visíveis somente sob lente de dez vezes, não estratificados. Vasos visíveis somente sob lente de dez vezes, solitários em sua maioria. Camadas de crescimento indistintas.

Descrição microscópica: porosidade difusa, vasos solitários, ocorrendo também múltiplos de dois, três e quatro (Figura 2C); diâmetro tangencial de 137,33 a 585,68 $\mu \mathrm{m}$; forma da seção arredondada, pontoações parenquimovasculares alternas, até sete vasos por $\mathrm{mm}^{2}$; elementos vasculares variando de 113,20 a 476,50 $\mu \mathrm{m}$ de comprimento (Tabela 2). Fibras (Figura 3C) com pontoações simples, não septadas, com paredes muito finas; variando de 440,73 a 902,36 $\mu \mathrm{m}$ de comprimento; diâmetro do lume de 10 até $17,39 \mu \mathrm{m}$; espessura da parede variando de 2,24 a 5,87 $\mu \mathrm{m}$ (Tabela 3). Parênquima axial paratraqueal aliforme e vasicêntrico. Raios não estratificados e homogêneos, constituídos de células procumbentes (Figura 4C), apresentando altura em número de células de 9 a 16 e 118,56 a 258,92 $\mu \mathrm{m}$ de altura; largura em

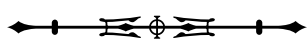


número de células 1 a 2, sendo $88 \%$ unisseriado e 17,45 a 27,35 $\mu \mathrm{m}$ de largura; número de raios por milímetro linear variando de 8 a 14 raios (Tabela 4).

\section{TACHIGALI PARAENSIS (HUBER) BARNEBY}

Descrição macroscópica (Figura 1D): parênquima axial contrastado, visível a olho nu, vasicêntrico e aliforme de aletas curtas, formando arranjos diagonais. Raios visíveis a olho nu, não estratificados. Vasos visíveis somente sob lente de dez vezes, solitários em sua maioria. Camadas de crescimento indistintas.

Descrição microscópica: porosidade difusa, vasos solitários, ocorrendo também múltiplos de dois, três e quatro (Figura 2D); diâmetro tangencial variando de 156,20 a 313,80 $\mu \mathrm{m}$; forma da seção arredondada, pontoações parênquimo-vasculares alternas, até seis vasos por mm²; elementos vasculares de 220,80 a 485,30 $\mu \mathrm{m}$ de comprimento (Tabela 2). Fibras (Figura 3D) com pontoações simples, não septadas, com paredes finas, variando de 631,50 a 1.402,3 $\mu \mathrm{m}$ de comprimento; diâmetro do lume de 9,45 a 33,93 $\mu \mathrm{m}$; espessura da parede de 2,58 a 5,26 $\mu \mathrm{m}$ (Tabela 3). Parênquima axial paratraqueal vasicêntrico e aliforme de aletas curtas. Raios não estratificados e homogêneos, constituídos de células procumbentes (Figura 4D), apresentando altura em número de células de 8 a 20, e variando de 127,55 a 406,73 $\mu \mathrm{m}$ de altura; largura em número de células 1 , sendo $100 \%$ unisseriado, variando de 7,54 a $30,15 \mu \mathrm{m}$ de largura; número de raios por milímetro linear variando de 9 a 15 raios (Tabela 4 ).

\section{TACHIGALI RETICULOSA (DWYER) ZARUCCHI \& HEREND.}

Descrição macroscópica (Figura 1E): parênquima axial contrastado, visível somente sob lente de dez vezes, aliforme e vasicêntrico, formando arranjos diagonais. Raios visíveis somente sob lente de dez vezes, não estratificados. Vasos visíveis somente sob lente de dez vezes, múltiplos em sua maioria. Camadas de crescimento indistintas.
Descrição microscópica: porosidade difusa, vasos múltiplos, ocorrendo também solitários (Figura 2E); diâmetro tangencial de 120,71 a 231,85 $\mu \mathrm{m}$; forma da seção arredondada, pontoações intervasculares alternas (Figura 5B); até 13 vasos por $\mathrm{mm}^{2}$, elementos vasculares de 229,0 a 466,1 $\mu \mathrm{m}$ de comprimento (Tabela 2). Fibras (Figura 3E) com pontoações simples, não septadas, com paredes muito finas; 580,18 a 1.022,71 $\mu$ m de comprimento; diâmetro do lume de 11,42 a 19,89 $\mu \mathrm{m}$; espessura da parede de 2,14 a 4,49 $\mu \mathrm{m}$ (Tabela 3). Parênquima axial paratraqueal aliforme e vasicêntrico. Raios não estratificados e homogêneos, constituídos de células procumbentes (Figura 4E), apresentando altura em número de células de 3 a 14, variando de 71,13 a 280,26 $\mu$ m de altura; largura em número de células 1-2, sendo $90 \%$ unisseriado, variando de 9,39 a 23,25 $\mu$ m de largura; número de raios por milímetro linear variando de 6 a 10 raios (Tabela 4).

\section{TACHIGALI SETIFERA (DUCKE) ZARUCCHI \& HEREND}

Descrição macroscópica: parênquima axial contrastado, visível somente sob lente de 10 vezes, aliforme e vasicêntrico, formando arranjos diagonais (Figura 1F). Raios visíveis a olho nu, não estratificados. Vasos visíveis somente sob lente de dez vezes, solitários em sua maioria. Camadas de crescimento indistintas.

Descrição microscópica: porosidade difusa, vasos solitários, ocorrendo também múltiplos de dois, três e quatro (Figura 2F); diâmetro tangencial de 118,48 a 416,97 $\mu \mathrm{m}$; forma da seção arredondada, pontoações parênquimo-vasculares alternas (Figura 5A), possuindo também pontoações intervasculares alternas (Figura 5E), até seis vasos por $\mathrm{mm}^{2}$; elementos vasculares variando de 214,33 a 550,25 $\mu \mathrm{m}$ de comprimento (Tabela 2). Fibras (Figura 3F) com pontoações simples, não septadas, com paredes muito finas, variando de 644,20 a 1220,52 $\mu \mathrm{m}$ de comprimento; diâmetro do lume de 7,10 a 17,72 $\mu \mathrm{m}$; espessura da parede de 2,41 a 5,08 $\mu \mathrm{m}$ (Tabela 3). Parênquima axial paratraqueal aliforme e vasicêntrico. 
Raios não estratificados e homogêneos, constituídos de células procumbentes (Figura 4F), apresentando altura em número de células de 2 a 15, variando de 74,42 a 215,55 $\mu \mathrm{m}$ de altura; largura em número de células 1 , sendo $100 \%$ unisseriado, variando de 8,25 a $26,80 \mu \mathrm{m}$ de largura; número de raios por milímetro linear variando de 6 a 12 raios (Tabela 4).

\section{TACHIGALI TINCTORIA BENTH. VAR. TINCTORIA (BENTH.) ZARUCCHI \& HEREND.}

Descrição macroscópica (Figura 1G): parênquima axial contrastado, visível somente sob lente de dez vezes, escasso e vasicêntrico, sem arranjos. Raios visíveis somente sob lente de dez vezes, não estratificados. Vasos visíveis somente sob lente de dez vezes, solitários em sua maioria. Camadas de crescimento indistintas.

Descrição microscópica: porosidade difusa, vasos solitários, ocorrendo também múltiplos de dois, três e quatro (Figura 2G); diâmetro tangencial de 118,90 a 267,30 $\mu \mathrm{m}$; forma da seção arredondada, pontoações parênquimovasculares alternas, até seis vasos por $\mathrm{mm}^{2}$; elementos vasculares de 229,70 a 428,30 $\mu \mathrm{m}$ de comprimento (Tabela 2). Fibras (Figura 3G) com pontoações simples, não septadas, com paredes muito finas, variando de 501,22 a 960,11 $\mu \mathrm{m}$ de comprimento; diâmetro do lume de 11,42 a 21,67 $\mu \mathrm{m}$; espessura da parede de 1,00 a 4,74 $\mu \mathrm{m}$ (Tabela 3). Parênquima axial paratraqueal, escasso e vasicêntrico. Raios não estratificados e homogêneos, constituídos de células procumbentes (Figura 4G), apresentando altura em número de células 3-10, variando de 70,60 a 194,30 $\mu \mathrm{m}$ de altura; largura em número de células 1 , sendo 100\% unisseriado, variando de 10,10 a 20,20 $\mu \mathrm{m}$ de largura; número de raios por milímetro linear variando de 6 a 11 raios (Tabela 4).

\section{DISCUSSÃO}

A caracterização anatômica das espécies estudadas corrobora com o que é mencionado para a família: placas de perfuração simples, pontoações alternas, parênquima paratraqueal em diferentes tipos e raios algumas vezes homocelulares (Baas et al., 2000; Metcalfe \& Chalk, 1950; Wheeler et al., 2007; Marcati et al., 2001).

Apenas T. froesii apresentou camadas de crescimento, sendo estas individualizadas por zonas fibrosas tangenciais mais escuras. Segundo Lima et al. (2009) e Wheeler et al. (2007), a ocorrência de camadas de crescimento em espécies de regiões tropicais são ocasionais.

Todas as espécies apresentaram vasos solitários, exceto $T$. reticulosa e $T$. melanocarpa, que possuem, em sua maioria, vasos múltiplos. Essas características estão de acordo com os resultados encontrados por Alves \& Angyalossy-Alfonso (2002) e Wheeler et al. (2007), os quais realizaram uma correlação das características anatômicas com diferentes habitats, citando ainda que estas características são muito frequentes em espécies lenhosas da flora brasileira ocorrentes em diversos ecossistemas. T. paniculata apresentou porosidade difusa, com grande parte dos vasos solitários, seguido de múltiplos de dois, vasos com placa de perfuração simples, parênquima paratraqueal vasicêntrico e raios homogêneos formados de células procumbentes; tais características já foram descritas em duas variedades de T. paniculata por Pires \& Marcati (2005) e publicado como Sclerolobium paniculatum Vog.

As espécies possuem também placa de perfuração simples e pontoações parênquimo-vasculares e intervasculares alternas, conforme verificado por Baas et al. (2000), Metcalfe \& Chalk (1950) e Pires \& Marcati (2005), estes últimos estudando duas variedades de Sclerolobium. Essas placas de perfuração e pontoações são consideradas características mais derivadas na linha evolutiva (Baas et al., 2000).

Todas as espécies apresentaram dois tipos de parênquima, sendo o paratraqueal vasicêntrico comum a todas as espécies. $\bigcirc$ parênquima axial vasicêntrico é característica da familia Fabaceae, e as variações podem ser utilizadas para distinção de espécie, tribo e subfamilias, o que jáfoi observado por Metcalfe \& Chalk (1950), Gasson (1999), Gasson et al. (2004), Pernía \& Melandri (2006) e Lima et al. (2009).

A presença de cristais prismáticos foi identificada somente nas células procumbentes dos raios em T. froesii. 

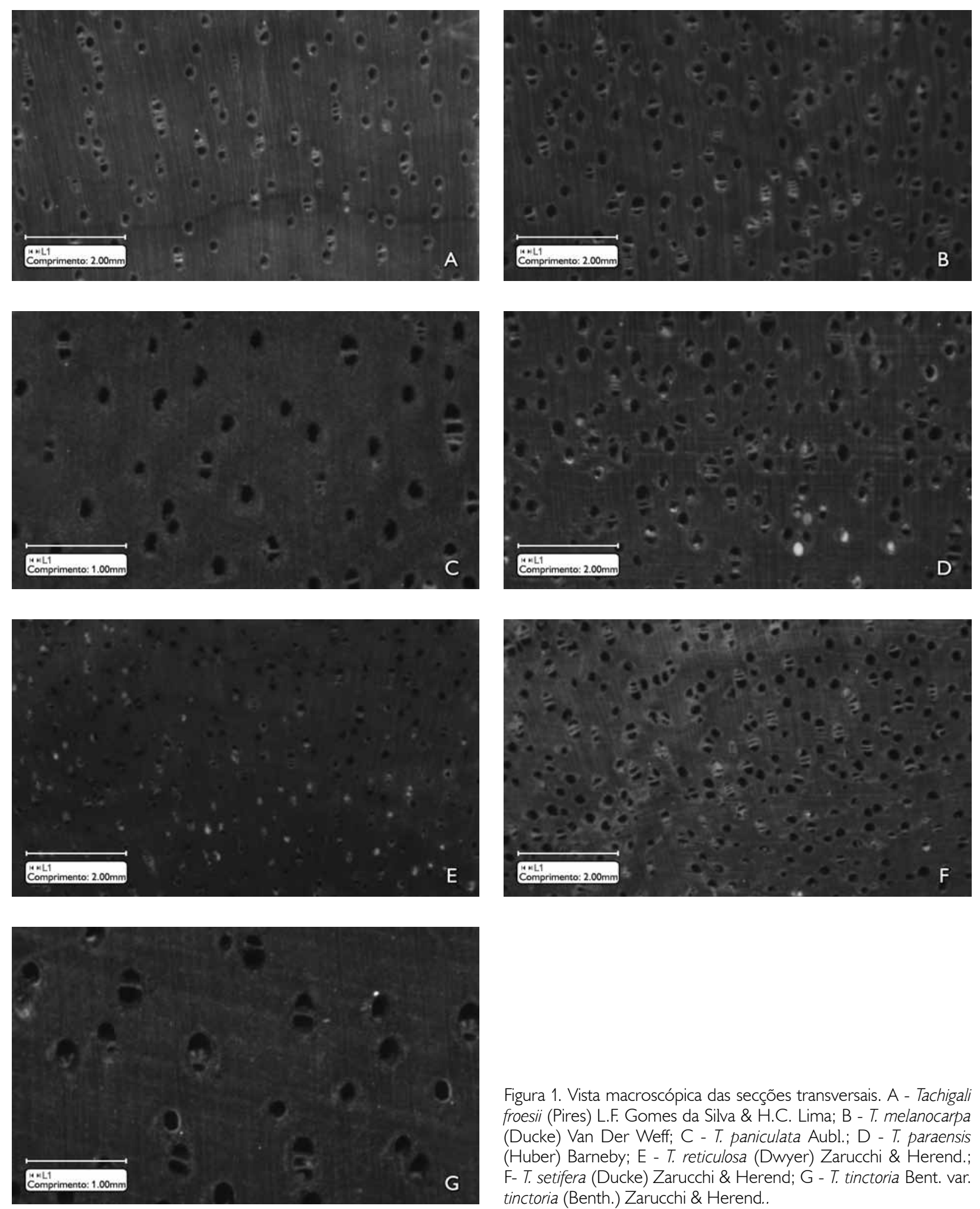

Figura 1. Vista macroscópica das secções transversais. A - Tachigali froesii (Pires) L.F. Gomes da Silva \& H.C. Lima; B - T. melanocarpa (Ducke) Van Der Weff; C - T. paniculata Aubl.; D - T. paraensis (Huber) Barneby; E - T. reticulosa (Dwyer) Zarucchi \& Herend.; F- T. setifera (Ducke) Zarucchi \& Herend; G - T. tinctoria Bent. var. tinctoria (Benth.) Zarucchi \& Herend. 

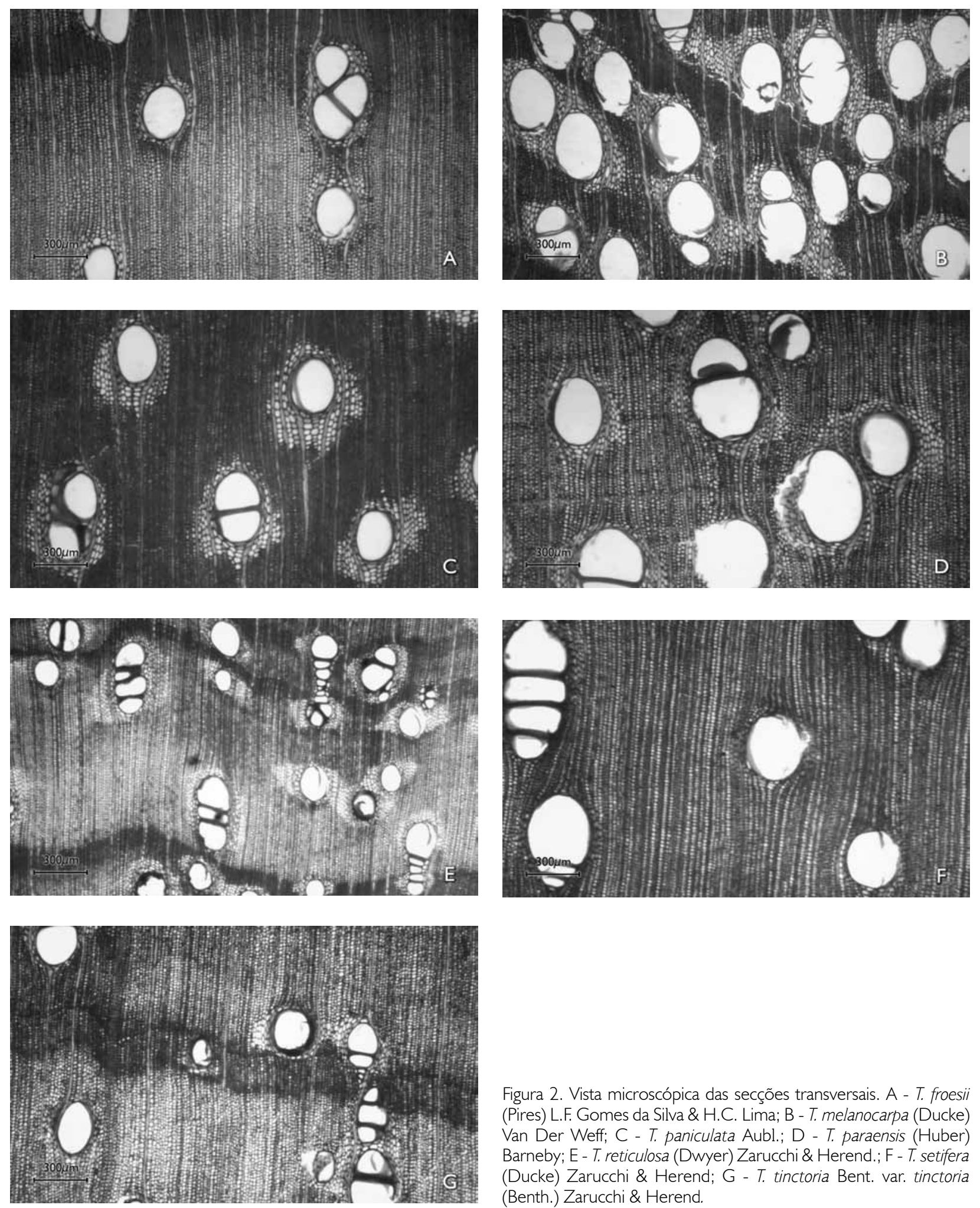

Figura 2. Vista microscópica das secções transversais. A - T. froesii (Pires) L.F. Gomes da Silva \& H.C. Lima; B - T. melanocarpa (Ducke) Van Der Weff; C - T. paniculata Aubl.; D - T. paraensis (Huber) Barneby; E - T. reticulosa (Dwyer) Zarucchi \& Herend.; F - T. setifera (Ducke) Zarucchi \& Herend; G - T. tinctoria Bent. var. tinctoria (Benth.) Zarucchi \& Herend.

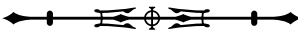



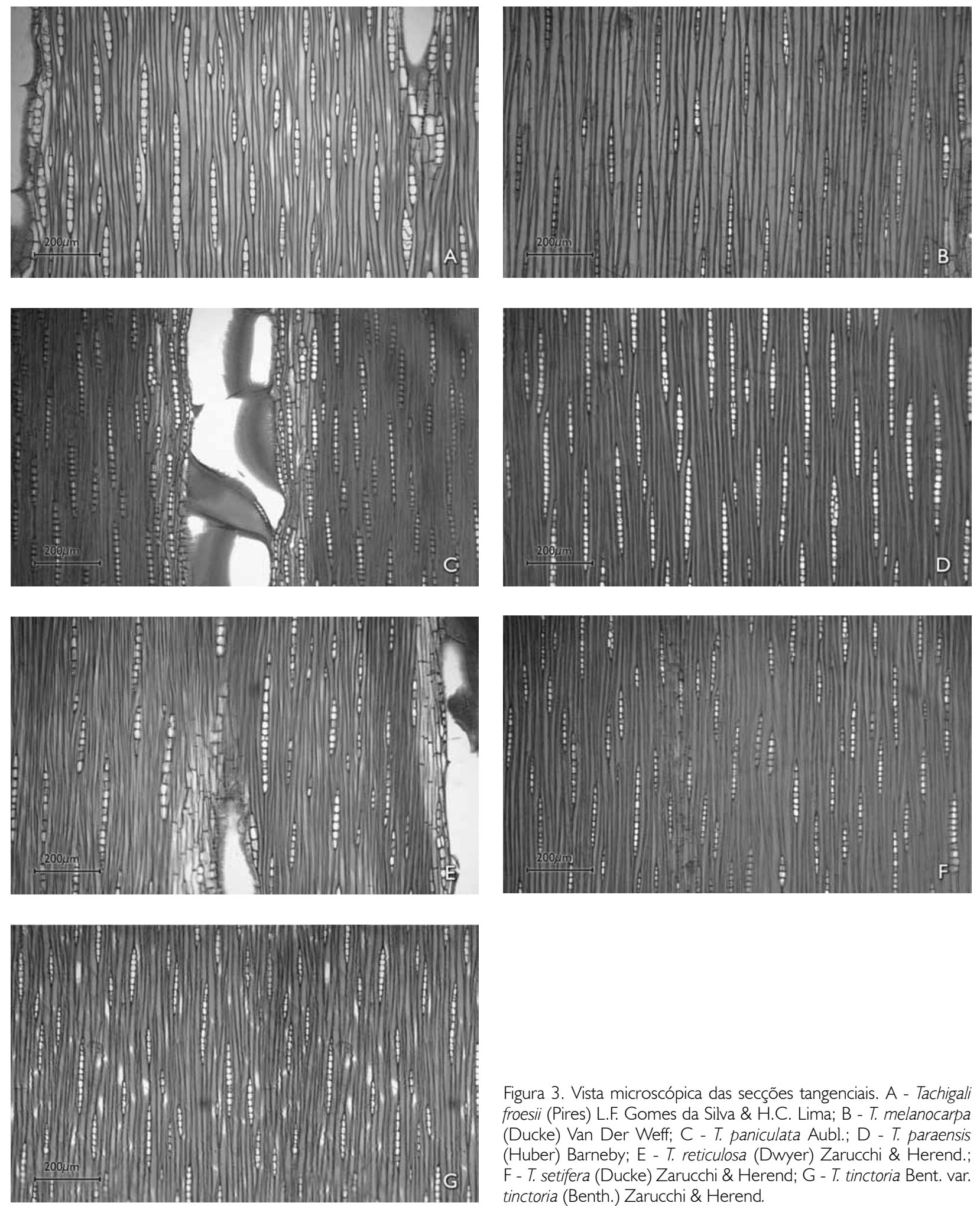

Figura 3. Vista microscópica das secções tangenciais. A - Tachigali froesii (Pires) L.F. Gomes da Silva \& H.C. Lima; B - T. melanocarpa (Ducke) Van Der Weff; C - T. paniculata Aubl.; D - T. paraensis (Huber) Barneby; E - T. reticulosa (Dwyer) Zarucchi \& Herend.; F - T. setifera (Ducke) Zarucchi \& Herend; G - T. tinctoria Bent. var. tinctoria (Benth.) Zarucchi \& Herend.

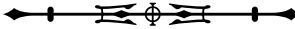



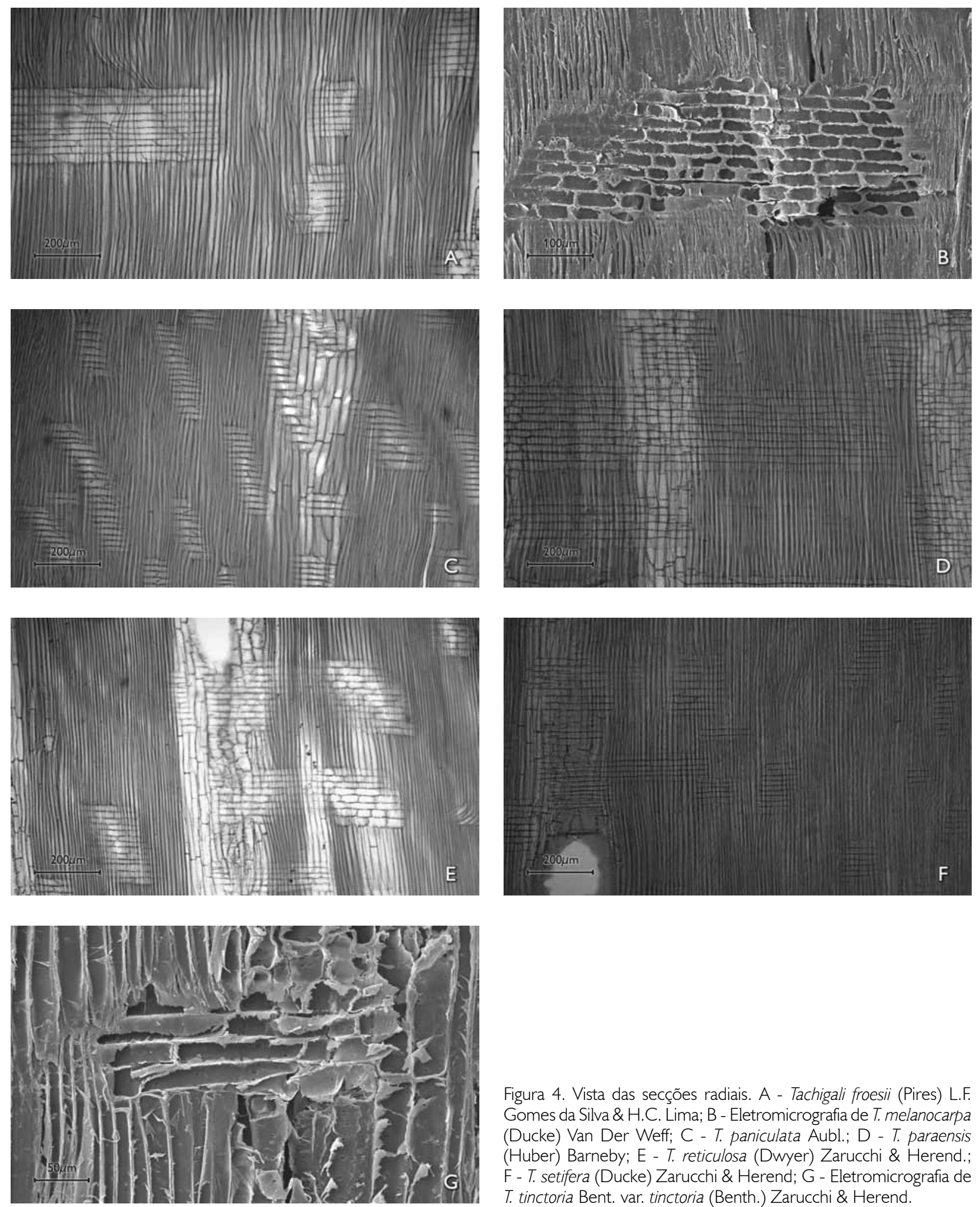

Figura 4. Vista das secções radiais. A - Tachigali froesii (Pires) L.F. Gomes da Silva \& H.C. Lima; B - Eletromicrografia de T. melanocarpa (Ducke) Van Der Weff; C - T. paniculata Aubl.; D - T. paraensis (Huber) Barneby; E - T. reticulosa (Dwyer) Zarucchi \& Herend.; F - T. setifera (Ducke) Zarucchi \& Herend; G - Eletromicrografia de T. tinctoria Bent. var. tinctoria (Benth.) Zarucchi \& Herend.

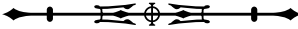



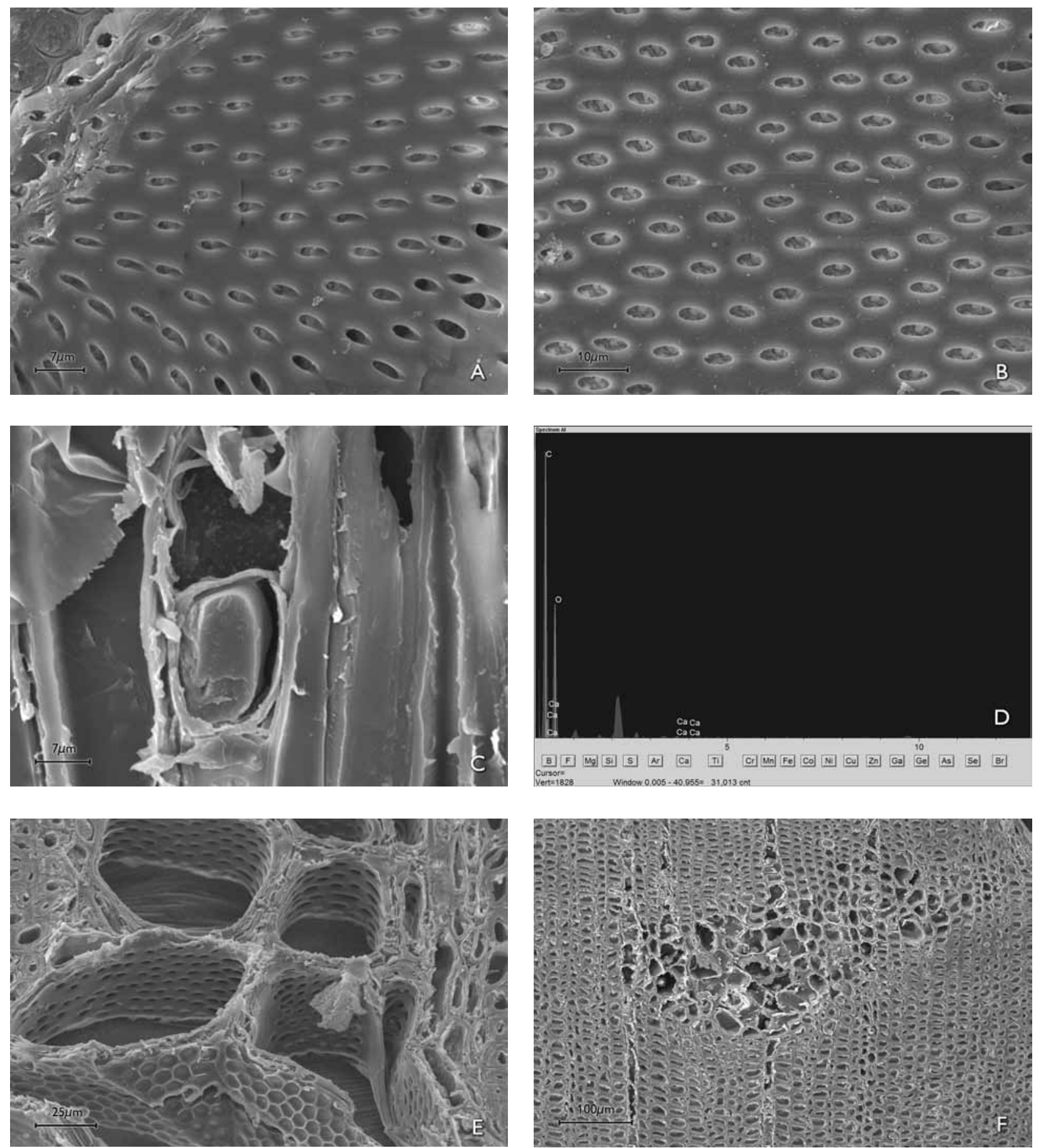

Figura 5. Detalhe das estruturas encontradas em algumas espécies estudadas. A - Eletromicrografia das pontoações parênquimo-vasculares alternas de Tachigali setifera (Ducke) Zarucchi \& Herend; B - Eletromicrografia das pontoações intervasculares alternas de T. reticulosa (Dwyer) Zarucchi \& Herend.; C - Eletromicrografia do cristal prismático de carbonato de cálcio de T. froesii (Pires) L.F. Gomes da Silva \& H.C. Lima; D - Espectro da microanálise química, em EDS/MEV, dos cristais de carbonato de cálcio observados na Figura 5C; E - Eletromicrografia das pontoações intervasculares alternas de T. setifera; F - Eletromicrografia do floema incluso T. froesii. 
Tais cristais são compostos por carbonato de cálcio, confirmado pelo EDS. Segundo IAWA Committee (1989), Vasconcelos et al. (1993; 1995) e Silva et al. (2001), a presença de cristais na madeira de espécies tropicais é comum, sendo utilizados na classificação e distinção de espécies, de acordo com a quantidade, tamanho e distribuição. Cristais de oxalato de cálcio foram observados em espécies de acácia por Gourlay \& Grime (1994). T. froesii foi a única espécie que apresentou floema incluso, podendo ser facilmente diferenciada das demais espécies em nível microscópico por este caráter. Não foram referenciados este padrão de distribuição do floema e essas estruturas para outra espécie da família. Esse é o primeiro registro de ocorrência de floema incluso em espécies da família.

Verificou-se que todas as espécies apresentaram fibras com pontoações simples, não septadas, semelhante aos resultados de Vatairea paraensis Ducke em estudos realizados por Ferreira et al. (2004). Tachigali paniculata possui fibras de menor comprimento entre as sete espécies estudadas e Tachigali paraensis, fibras de maior comprimento. As fibras são os elementos celulares constituintes da madeira de maior importância do ponto de vista tecnológico; por esta razão, o conhecimento da quantidade desses elementos e de sua estrutura é de fundamental importância para a qualificação de madeiras para atividades diversas (Paula, 2003).

\section{CONCLUSÕES}

Tachigali froesii foi a espécie que mais apresentou estruturas que a diferenciam das demais, como floema incluso e cristais de carbonato de cálcio nas células procumbentes dos raios, além da heterogeneidade dos raios. Este trabalho é o primeiro registro de floema incluso em uma espécie da família. Pode ser observado que parênquima axial paratraqueal vasicêntrico é característico da familia. Para que sejam delimitadas as espécies no presente estudo, é necessário reunir um conjunto de características, sendo as principais os tipos de parênquimas axiais presentes e a composição das células dos raios, no plano longitudinal radial.

\section{AGRADECIMENTOS}

Os autores agradecem ao Museu Paraense Emílio Goeldi e ao Conselho Nacional de Desenvolvimento Científico e Tecnológico (CNPq).

\section{REFERÊNCIAS}

ALVES, E. S. \& V. ANGYALOSSY-ALFONSO, 2002. Ecological trends in the wood anatomy of some Brazilian species. 2. Axial parenchyma, rays and fibers. IAWA Journal 23: 391-418.

ANGYALOSSY-ALFONSO, V. \& R. B. MILLER, 2002. Wood anatomy of the Brazilian species of Swartzia and considerations within the tribe Swartzieae. IAWA Journal 23(4): 359-390.

BAAS, P., E. WHELLER \& M. CHASE, 2000. Dycotyledonous wood anatomy and the APG system of angiosperm classification. Botanical Journal of the Linnean Society 131: 3-17.

BARETA-KUIPERS, T., 1981. Wood anatomy of Leguminosae: its relevance to taxonomy: 677-705. In: R. M. POLHILL \& P. A. RAVEN (Eds.): Advance in legume systematics. Royal Botanic Gardens, Kew.

CORADIN, V. T. R. \& G. I. B. MUNIZ, 1992. Normas para procedimentos em estudos de anatomia de madeira: I. Angiospermae, II. Gimnospermae: 1-19. IBAMA, LPF (Série Técnica, 15), Brasilia.

FERREIRA, G. C. \& M. J. G. HOPKINS, 2004. Manual de identificação botânica e anatômica - angelim: 1-101. Embrapa Amazônia Oriental, Belém.

FERREIRA, G. C., J. L. GOMES \& M. J. G. HOPKINS, 2004. Estudo anatômico das espécies de leguminosae comercializadas no estado do Pará como "angelim". Acta Amazonica 34(3): 387-398.

FRANKLIN, G. L., 1945. Preparation of thin sections of synthetic resins and wood - resin composites, and a new macerating method for wood. Nature 155(3924): 51.

GASSON, P., 1999. Wood anatomy of the tribe Dipterygeae with comments on related Papilionoid and Caesalpinioid Leguminosae. IAWA Journal 20(4): 441-455.

GASSON, P. \& E. J. WRAY, 2001. Wood anatomy of Cyathostegia mathewsii (Swartzieae, Papilionoideae, Leguminosae). IAWA Journal 22(2): 193-199.

GASSON, P., E. WRAY \& B. D. SCHRIRE, 2004. Wood anatomy of the tribe Millettieae with comments on related Papilionoid Leguminosae. IAWA Journal 25(4): 485-545.

GRAHAM, A. \& G. BARKER, 1981. Palynology and tribal classification in the Caesalpinioideae: 801-834. In: R. M. POLHILL \& P. H. RAVEN (Eds.): Advances in legume systematics. Royal Botanic Gardens, Kew. 
GOURLAY, I. D. \& G. W. GRIME, 1994. Calcium oxalate crystals in African acacia species and their analysis by scanning proton microprobe (SPM). IAWA Journal 25(2): 137-148.

HASTON, E. M., G. P. LEWIS \&J. A. HAWINS, 2003. A phylogenetic investigation of the Peltophorum group (Caesalpinieae: Leguminosae): 149-159. In: B. B. KLITGAARD \& A. BRUNEAU (Ed.): Advances in legume systematics. Royal Botanic Gardens, Kew.

HASTON, E. M., G. P. LEWIS \& J. A. HAWKINS, 2005. A phylogenetic reappraisal of the Peltophorum group (Caesalpinieae: Leguminosae) based on the chloroplast trnL-F, rbcL and $r p s 16$ sequence data. American Journal of Botany 92(8): 1359-1371.

HÖHN, A., 1999. Wood anatomy of selected West African species of Caesalpinioideae and Mimosoideae (Leguminosae): a comparative study. IAWA Journal 20(2): 115-146.

IAWA COMMITTEE, 1989. International Association of Wood Anatomists. List of microscope features for hardwood identification. IAWA Bulletin 10(3): 234-332

LEWIS, G. P., B. SCHRIRE, B. MACKINDER \& M. LOCK, 2005. Legumes of the world. Royal Botanic Gardens, Kew.

LIMA, R. S., P. OLIVEIRA \& L. R. RODRIGUES, 2009. Anatomia do lenho de Enterolobium contortisiliquum (Vell.) Morong (LeguminosaeMimosoideae) ocorrente em dois ambientes. Revista Brasileira de Botânica 32: 361-374.

LOUREIRO, A. A., F. J. VASCONCELLOS \& J. A. FREITAS, 1983. Contribuição ao estudo anatômico do lenho de 5 espécies de Sclerolobium e 5 espécies de Tachigalia (Leguminosa) da Amazônia. Acta Amazonica 13(1): 149-170.

LOUREIRO, A. A., J. A. FREITAS, K. B. L. RAMOS \& C. A. A. FREITAS, 1997. Essências madeireiras da Amazônia: v. 3: 1-103. MCT/INPA-CPPF, Manaus.

LOUREIRO, A. A., J. A. FREITAS, K. B. L. RAMOS \& C. A. A. FREITAS, 2000. Essências madeireiras da Amazônia: v. 4: 1-191. MCT/INPA-CPPF, Manaus.

MARCATI, C. R., V. ANGYALOSSY-ALFONSO \& L. BENETATI, 2001. Anatomia comparada do lenho de Copaifera langsdorffii Desf. (Leguminosae-Caesalpinoideae) de floresta e cerradão. Revista Brasileira de Botânica 24: 311-320.

MARCHIORI, J. N. C., 2007. Dendrologia das angiospermas: leguminosas. $2^{\text {nd }}$ ed.: 1-199. UFSM, Santa Maria.
METCALFE, C. R. \& L. CHALK, 1950. Anatomy of the Dicotyledons: 1: 1-724. Claredon Press, Oxford.

OLIVEIRA, E., B. R. VITAL, A. S. PIMENTA, R. M. D. LUCIA, A. M. M. LADEIRA \& A. C. O. CARNEIRO, 2006. Estrutura anatômica da madeira e qualidade do carvão de Mimosa tenuiflora (Willd.) Poir. Revista Árvore 30(2): 311-318.

PAULA, J. E., 2003. Caracterização anatômica da madeira de sete espécies da Amazônia com vistas à produção de energia e papel. Acta Amazonica 33(2): 243-262.

PERNÍA, N. E. \& J. L. MELANDRI, 2006. Wood anatomy of the tribe Caesalpinieae (Leguminosae, Caesalpinioideae) in Venezuela. IAWA Journal 27(1): 99-114.

PIRES, I. P. \& C. R. MARCATI, 2005. Anatomia e uso da madeira de duas variedades de Sclerolobium paniculatum Vog. do sul do Maranhão, Brasil. Acta Botanica Brasilica 19(4): 669-678.

SILVA, L. B., F. A. R. SANTOS, P. GASSON \& D. CUTLER, 2009. Anatomia e densidade básica da madeira de Caesalpinia pyramidalis Tul. (Fabaceae), espécie endêmica da caatinga do Nordeste do Brasil. Acta Botanica Brasilica 23(2): 436-445.

SILVA, L. F. G. \& H. C. LIMA, 2007. Mudanças nomenclaturais no gênero Tachigali aubl. (Leguminosae - Caesalpinioideae) no Brasil. Rodriguésia 58(2): 397-401.

SILVA, J. N. M., J. O. P. CARVALHO \& J. A. G. YARED, 2001. A silvicultura na Amazônia Oriental: contribuição do projeto. EMBRAPA/DFID, Belém.

SILVEIRA, M., 1989. Preparação de amostras biológicas para microscopia eletrônica de varredura. In: W. SOUZA (Ed.): Manual sobre técnicas básicas em microscopia eletrônica: 71-79. USP, São Paulo.

VASCONCELOS, F. J., A. C. SILVA \& J. A. FREITAS, 1993. Deposição de sílica e cristais no xilema de espécies tropicais da família Caesalpiniaceae. Revista Árvore 17(3): 369-374.

VASCONCELOS, F. J., J. A. FREITAS \& A. C. SILVA, 1995. Observações microscópicas de inclusões minerais no xilema de espécies tropicas na Amazônia. Acta Amazonica 25(1/2): 55-68.

WHEELER, E. A., P. BAAS \& S. RODGERS, 2007. Variations in dicot wood anatomy: a global analysis based on the insidewood database. IAWA Journal 28: 229-259.

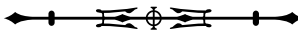


